\title{
Chronic Eccentric Cycling Improves Quadriceps Muscle Structure and Maximum Cycling Power
}

Authors

Affiliations

\section{H. Leong ', W. J. McDermott ${ }^{2}$, S. J. Elmer ${ }^{3,4}$, J. C. Martin}

Department of Exercise and Sport Science, University of Utah, Salt Lake City, Utah, United States Sport Science and Research, The Orthopedic Specialty Hospital, Murray, Utah, United States Department of Exercise Science and STEM, University of Maine, Orono, Maine, United States

Department of Mechanical Engineering, University of Maine, Orono, Maine, United States
Key words

eccentric muscle contraction

strength training

force suppression

quadriceps hypertrophy

muscular power accepted after revision September 11, 2013

Bibliography DOI http://dx.doi.org/ 10.1055/s-0033-1358471 Published online: November 14, 2013 Int J Sports Med 2014; 35 : 559-565 (c) Georg Thieme Verlag KG Stuttgart · New York ISSN 0172-4622

\section{Correspondence}

Chee Hoi Leong

Exercise and Sport Science University of Utah College of Health 84112 Salt Lake City United States Tel.: + 1/801/5815430 Fax: + 1/801/585 3992 cheehoi.leong@utah.edu

\section{Abstract} $\nabla$

An interesting finding from eccentric exercise training interventions is the presence of muscle hypertrophy without changes in maximum concentric strength and/or power. The lack of improvements in concentric strength and/or power could be due to long lasting suppressive effects on muscle force production following eccentric training. Thus, improvements in concentric strength and/or power might not be detected until muscle tissue has recovered (e.g., several weeks post-training). We evaluated alterations in muscular structure (rectus-femoris, RF, and vastus lateralis, VL, thickness and pennation angles) and maximum concentric cycling power $\left(\mathrm{P}_{\max }\right)$ 1-week following 8-weeks of eccentric cycling

\section{Introduction}

$\nabla$

Eccentric exercise is a popular strength training paradigm. Investigators who have implemented eccentric training programs in healthy individuals generally report significant hypertrophy in the active muscles [17,20,21,29], as well as increased maximum eccentric and isometric strength $[21,22]$ following training. Results for maximum concentric strength and/or power, however, have been mixed with some investigators reporting increases [4,20], but most investigators have reported unchanged maximum concentric strength and/or power $[8,17,21,34]$. These mixed reports on maximum concentric strength and/or power improvements following eccentric training are curious because muscle force is generally a function of muscle excitation and cross-sectional area $[13,15]$. Furthermore, skeletal muscle hypertrophy with changes in maximum eccentric but not concentric strength and/or power implies that improvements are contraction or velocity-specific. Although such a unique finding could be due training (2×/week; 5-10.5 min; $20-55 \%$ of $\left.\mathrm{P}_{\max }\right)$. $\mathrm{P}_{\max }$ was assessed again at 8 -weeks post-training. At 1 week post-training, RF and VL thickness increased by $24 \pm 4 \%$ and $13 \pm 2 \%$, respectively, and RF and VL pennation angles increased by $31 \pm 4 \%$ and $13 \pm 1 \%$, respectively (all $P<0.05$ ). Compared to pre-training values, $\mathrm{P}_{\max }$ increased by $5 \pm 1 \%$ and $9 \pm 2 \%$ at 1 and 8 weeks post-training, respectively (both $P<0.05$ ). These results demonstrate that short-duration high-intensity eccentric cycling can be a time-effective intervention for improving muscular structure and function in the lower body of healthy individuals. The larger $\mathrm{P}_{\max }$ increase detected at 8-weeks post-training implies that sufficient recovery might be necessary to fully detect changes in muscular power after eccentric cycling training. to adaptations that are truly velocity-specific [36], this finding is not universal [32]. Alternatively, a suppressive effect associated with chronic eccentric training intervention may explain the lack of improvements in maximum concentric strength and/or power found in immediate post-training evaluations. In other words, eccentric exercise could suppress muscle force [11] and/or alter the neural control of muscle force [7]. Depending on the eccentric training intensity and duration, improvement in maximum concentric strength and/or power might not be fully realized until muscle tissue, neuromuscular control and reflex sensitivity has fully recovered or adapted (i.e., several weeks following end of training) $[2,6,37]$. Measures of concentric strength and/or power performed weeks after cessation of training may reflect fully adapted and recovered neuromuscular function. Such measures could support and expound upon previous findings of improved concentric strength and/or power.

Cycling provides a particularly effective model for evaluating the effects of eccentric training on 
maximal concentric function. That is, eccentric cycling can serve as repetitive, high-force, low-metabolic cost, multi-joint eccentric training modality which is known to produce significant hypertrophy $[16,17,29]$. Maximal concentric cycling can also be used to assess pre- to-post changes in maximum power $[8,17]$, thus providing similar actions for training and assessment. Accordingly, our purpose for conducting this investigation was to evaluate changes in muscle structure (rectus-femoris (RF) and vastus-lateralis (VL) thickness and pennation angles) and muscular function (maximum concentric cycling power, $\mathrm{P}_{\max }$ ) at 1 and 8 weeks following chronic eccentric cycling training. The timing of these measures would allow comparison with many previous studies in which pre- to post-changes in concentric strength and/or power were measured soon after training and provide sufficient time for recovery following chronic eccentric cycling training. We hypothesized that eccentric cycling training would induce structural changes to the muscle, which would be reflected in improved maximum concentric cycling power, with the largest increases occurring 8 weeks post-training.

\section{Methods}

$\nabla$

\section{Participants}

8 young healthy individuals ( 4 males and 4 females) volunteered to participate in this investigation (participant characteristics are presented in Table 1). Participants were instructed to refrain from strength training during eccentric cycling training and for the 8 weeks following the completion of the study. Experimental procedures used in this investigation were approved by the University of Utah Institutional Review Board and conformed to required ethical standards [18]. All participants provided written informed consent prior to engaging in experimental procedures.

\section{Experimental design}

The experimental protocol is described below and illustrated in Fig. 1. In this investigation, we implemented an 8-week eccentric cycling training intervention and evaluated pre- to post-training changes in muscle structure (RF and VL thickness and pennation angles) and maximum concentric cycling power

Table 1 Participant descriptive characteristics $(n=8)$.

\begin{tabular}{|lc|} 
& Mean \pm SD \\
\hline age (years) & $22 \pm 2$ \\
mass $(\mathrm{kg})$ & $69 \pm 13$ \\
\hline height $(\mathrm{m})$ & $1.7 \pm 0.1$ \\
BMI $\left(\mathrm{kg} / \mathrm{m}^{2}\right)$ & $23 \pm 2$ \\
\hline BMl: Body mass index & \\
\hline
\end{tabular}

$\left(P_{\max }\right) .1$ week before the start of the eccentric cycling training, participants reported to the laboratory for familiarization trials of maximal concentric cycling and for pre-training assessments of muscle structure and maximum cycling power. After these initial visits, participants performed eccentric cycling training 2 times per week for 8 consecutive weeks. 1 week after the final training session, participants returned to the laboratory where muscle structure and $\mathrm{P}_{\max }$ were assessed. Participants returned to the laboratory again 8 weeks after the final training session for the assessment of $\mathrm{P}_{\max }$. Participants also performed familiarization trials of maximal concentric cycling 1 week before the post-training and 8 weeks post-training measurements in order to retain any learning and to ensure that the participants were adept at the cycling technique [30].

\section{Eccentric cycling training}

Participants performed eccentric cycling training at $60 \mathrm{rpm}$ on an isokinetic eccentric cycling ergometer ( $\bullet$ Fig. 2) $[8,9]$. The progression of eccentric cycling training intensity and duration was modified from our previous work [8] and is summarized in - Table 2. Specifically, eccentric cycling power was initially set to $20 \%$ of pre-training $\mathrm{P}_{\max }$ for $5 \mathrm{~min}$ and progressively increased to $55 \%$ of pre-training $\mathrm{P}_{\max }$ for $10.5 \mathrm{~min}$ over the 8-week training period. Participants targeted the prescribed powers with feedback from a power meter (Schoberer Rad Messtechnik, SRM, Jülich, Germany). During the final minute of each training session, participants were asked to report ratings of perceived exertion for their total body exertion ( $\left(\mathrm{RE}_{\mathrm{body}}\right)$ as well their specific leg exertion ( $\mathrm{RPE}_{\text {legs }}$ ) using a Borg 6-20 scale (Borg, 1970). Heart rate was also was assessed during the final minute of every training session (Polar FT1, Kempele, Finland). Finally, prior to each eccentric cycling training session participants performed a bilateral squat movement during which they indicated the level of muscle soreness in their legs using a visual analog scale $(0-10 \mathrm{~cm})$ with $10 \mathrm{~cm}$ representing the worst pain imaginable [8]. A soreness value of less than $5 \mathrm{~cm}$ (representing moderate soreness) was used as an indication to proceed with subsequent eccentric cycling training. This measure was utilized to ensure that the eccentric cycling protocol was safely administered and tolerated by the uncompensated volunteer participants.

\section{Muscle structure}

Ultrasound imaging (LOGIQ e 2008, GE Healthcare, Wauwatosa, WI, USA) was used to examine muscle structure of RF and VL of the right quadriceps. Participants were placed in the supine position with knees resting comfortably in extension near the natural resting position of $10^{\circ}$ of flexion. Images of the RF were taken at the midpoint between the anterior superior iliac spine and the superior border of the patella. Vastus lateralis images were taken at the midpoint between the greater trochanter and

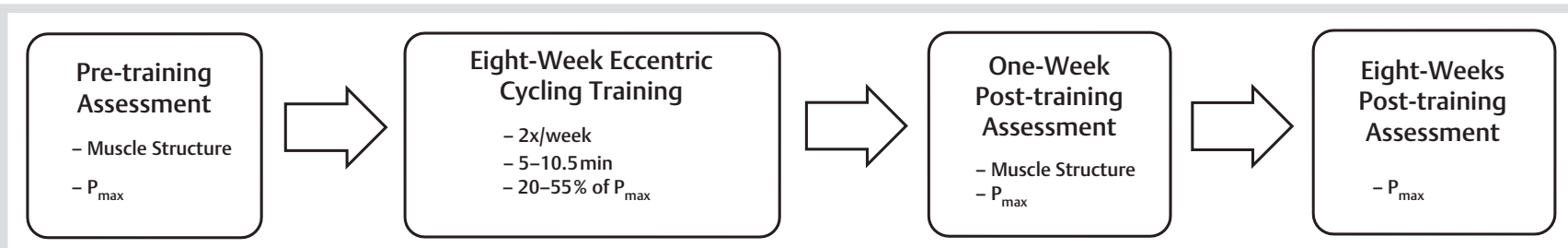

Fig. 1 Experimental protocol of the study. One week before the start of the eccentric cycling training, pre-training assessments of muscle structure and maximum concentric cycling power $\left(P_{\max }\right)$ were conducted. Next, participants performed eccentric cycling training 2 times per week for 8 consecutive weeks. One week after the final training session, muscle structure and $\mathrm{P}_{\max }$ were assessed. $\mathrm{P}_{\max }$ was assessed again 8 weeks after the final training session. 


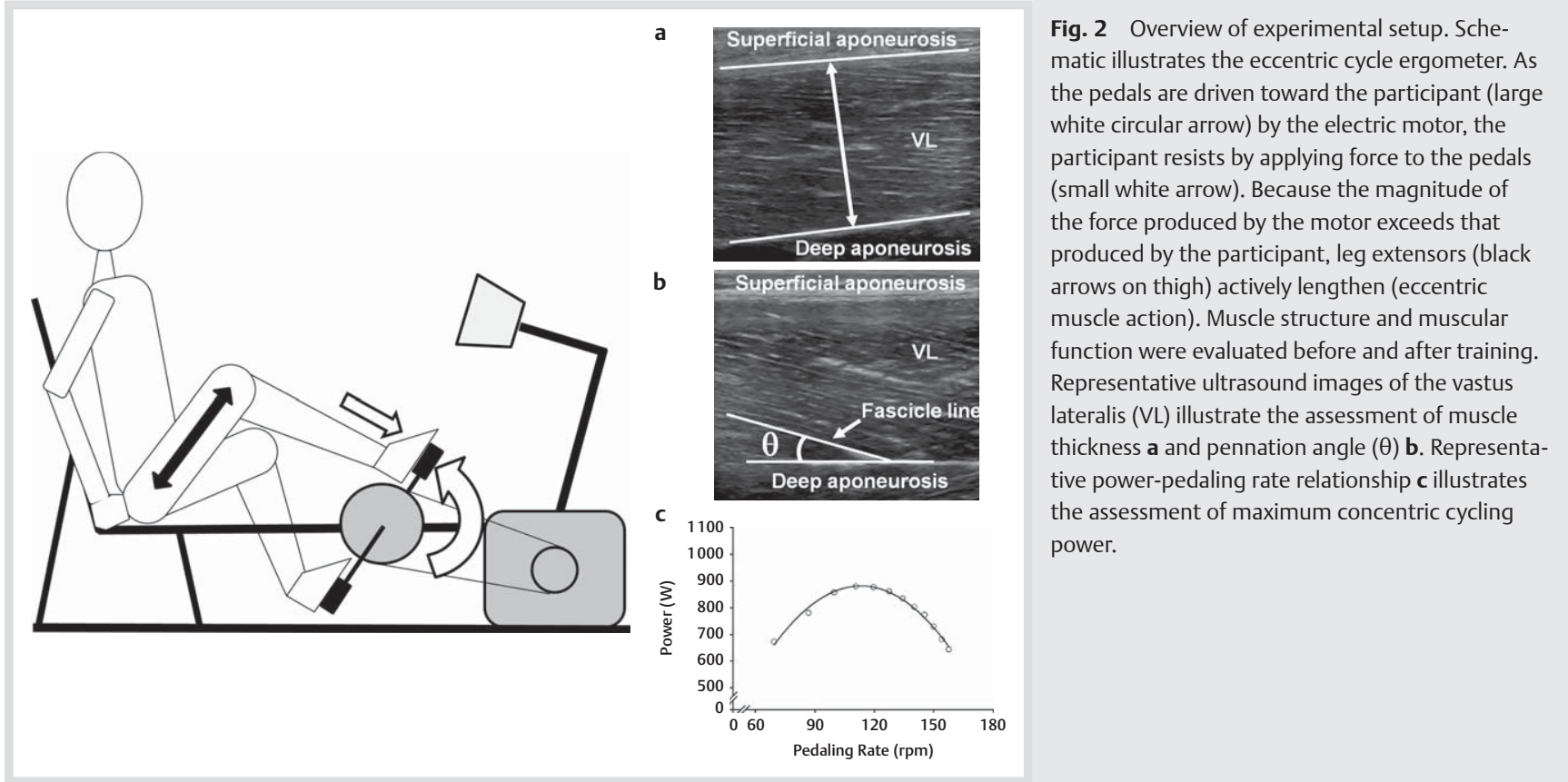

the lateral femoral epicondyle. Each midpoint was clearly marked on the skin to ensure proper placement of the probe during repeated scans. Electrode gel was applied to the skin to aid acoustic coupling and to eliminate compression or deformation of the muscle. Two-dimensional B-mode ultrasound imaging with a $6-12 \mathrm{MHz}$ linear array transducer was used to obtain images of the RF and VL. Digitizing software (ImageJ $1.46 \mathrm{r}$, National Institutes of Health, Bethesda, MD, USA) was used to assess (1) muscle thickness as the distance between the superficial and deep aponeuroses in the middle of the ultrasound image at a $90^{\circ}$ angle from the deep aponeurosis, and (2) pennation angle as the positive angle between the deep aponeurosis and the fascicle line.

\section{Maximum concentric cycling}

Participants performed maximal concentric cycling trials (4s) on an inertial-load cycle ergometer which measures maximal concentric cycling power across a range of pedaling rates (e.g., 60-180 rpm) in a single brief trial [31]. Following a 5-min cycling warm-up ( 50-125W), participants began each trial from rest and accelerated maximally for 8 pedal revolutions with resistance provided solely by the moment of inertia of the flywheel. Participants were instructed to remain seated throughout each trial and were given standardized verbal encouragement. Flywheel angular position data were low-pass filtered at $8 \mathrm{~Hz}$ using a $5^{\text {th }}$ order spline routine [38], and velocity and acceleration were determined from the spline coefficients. Power averaged over each complete crank revolution was calculated as rate of change in kinetic energy, and maximum power was identified as the apex of the power-pedaling rate relationship.

\section{Statistical analysis}

Paired student's t-tests were used to evaluate pre- to post-training changes in muscle thickness and pennation angle. A one-way repeated measures analysis of variance (ANOVA) and subsequent post hoc (Fisher least significant differences) analyses were used to evaluate pre- to post-training changes in $\mathrm{P}_{\max }$ (pre-training, 1 week and 8 weeks post-training). Effect sizes (ES) were calculated for all analyses and ES magnitudes of $0.10,0.30$, and 0.50 ,
Table 2 Progression of eccentric cycling training intensity and duration. Note that all training was performed at $60 \mathrm{rpm}$.

\begin{tabular}{|lcc|}
\hline Weeks of Training & \% Baseline $\mathbf{P}_{\mathbf{m a x}}$ & Duration (min) \\
\hline 1 & 20 & 5 \\
\hline 2 & 25 & 6 \\
3 & 30 & 7 \\
\hline 4 & 35 & 8 \\
\hline 5 & 40 & 9 \\
\hline 6 & 45 & 9.5 \\
7 & 50 & 10 \\
\hline 8 & 55 & 10.5 \\
\hline
\end{tabular}

were interpreted as small, medium, and large effects, respectively [12]. Values are reported as mean \pm SEM, and alpha was set at 0.05 .

\section{Results}

$\nabla$

All participants completed the 8 -week training study at $92 \pm 4 \%$ of prescribed training intensity. Average power absorption progressed from $157 \pm 24 \mathrm{~W}$ to $442 \pm 56 \mathrm{~W}$, and total work increased from $48 \pm 8 \mathrm{~kJ}$ to $272 \pm 35 \mathrm{~kJ}$ during the 8 -week training period ( $\bullet$ Fig. 3). Training heart rate, $\mathrm{RPE}_{\text {body, }}$ and $\mathrm{RPE}_{\text {legs }}$ also increased in response to the progressive eccentric cycling training ( $\bullet$ Fig. 3). Participants reported low $(0.6 \pm 0.2 \mathrm{~cm})$ to moderate $(2.5 \pm 0.9 \mathrm{~cm})$ levels of muscle soreness during the training period ( $\bullet$ Fig. 3 ).

Following eccentric cycling training RF and VL muscle thickness increased by $24 \pm 4 \%$ ( $18.6 \pm 1.3$ pre-training to $23.0 \pm 1.7 \mathrm{~mm}, 1$ week post-training) and $13 \pm 2 \%(19.6 \pm 1.4$ pre-training to $22.1 \pm 1.4 \mathrm{~mm}$, one week post-training), respectively, compared to pre-training values (both $P<0.01, E S=0.83$; $\odot$ Fig. 4). Similarly, RF and VL pennation angles increased by $31 \pm 4 \%$ (11.6 \pm 0.7 pre-training to $15.1 \pm 0.9^{\circ}$, 1 week post-training; $P<0.05, E S=$ $0.74)$ and $13 \pm 1 \%$ angle ( $15.8 \pm 1.0$ pre-training to $17.8 \pm 0.6^{\circ}, 1$ week post-training; $P<0.05, E S=0.70)$, respectively, compared to 

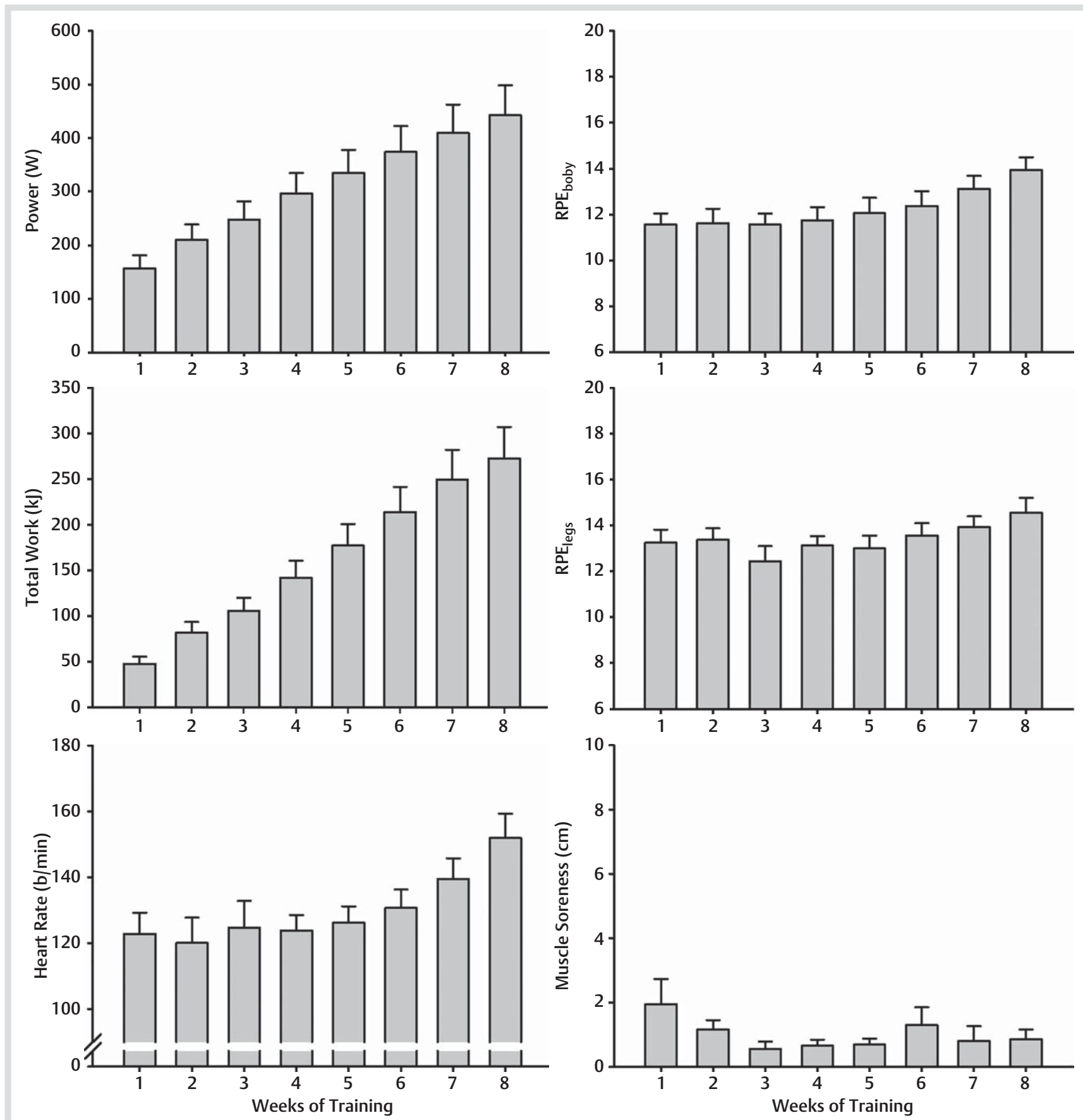

Fig. 3 Mechanical, cardiorespiratory and perceptual responses recorded during eccentric cycling training. Values are presented as mean \pm SEM.

pre-training values. Repeated measures ANOVA procedures revealed a significant main effect of time on $P_{\max }$ values $(P<0.01)$. Subsequent post-hoc analyses indicated that compared to pretraining values, $P_{\max }$ increased by $5 \pm 1 \%$ from $894 \pm 120 \mathrm{~W}$ pretraining to $941 \pm 124 \mathrm{~W}$ at 1 week post-training $(P<0.05, E S=0.78)$ and by $9 \pm 2 \%$ from $894 \pm 120 \mathrm{~W}$ pre-training to $970 \pm 128 \mathrm{~W}$ at 8 weeks post-training $\left(P<0.05, E S=0.82\right.$; $~$ Fig. 5). Although $\mathrm{P}_{\max }$ at 8-weeks post-training vs. 1 -week post-training was not statistically significant $(P=0.06, E S=0.64)$, the higher $P_{\max }$ observed in 11 out of the 12 participants at 8 week vs. 1 week post-training provides strong evidence for a trend of increasing $\mathrm{P}_{\max }$.

\section{Discussion}

$\nabla$

Our main finding in this study was that 8 weeks of eccentric cycling training elicited changes in both muscle thickness and pennation angle, and improved $\mathrm{P}_{\text {max }}$. Furthermore, the larger increase in $P_{\max }$ measured at 8 weeks post-training suggested that sufficient recovery following chronic eccentric cycling training might be necessary to fully detect changes in maximum concentric power. Thus, our intervention involving short-duration high-intensity eccentric cycling $(2 \times /$ week, $5-10.5 \mathrm{~min}$ at $20-55 \% P_{\max }$ ) served as a time-effective strategy for improving muscle structure and muscular function, and our post-testing period of 8 weeks allowed sufficient time for the recovery/adaptation. Although the gradual ramp-up protocol utilized in the 

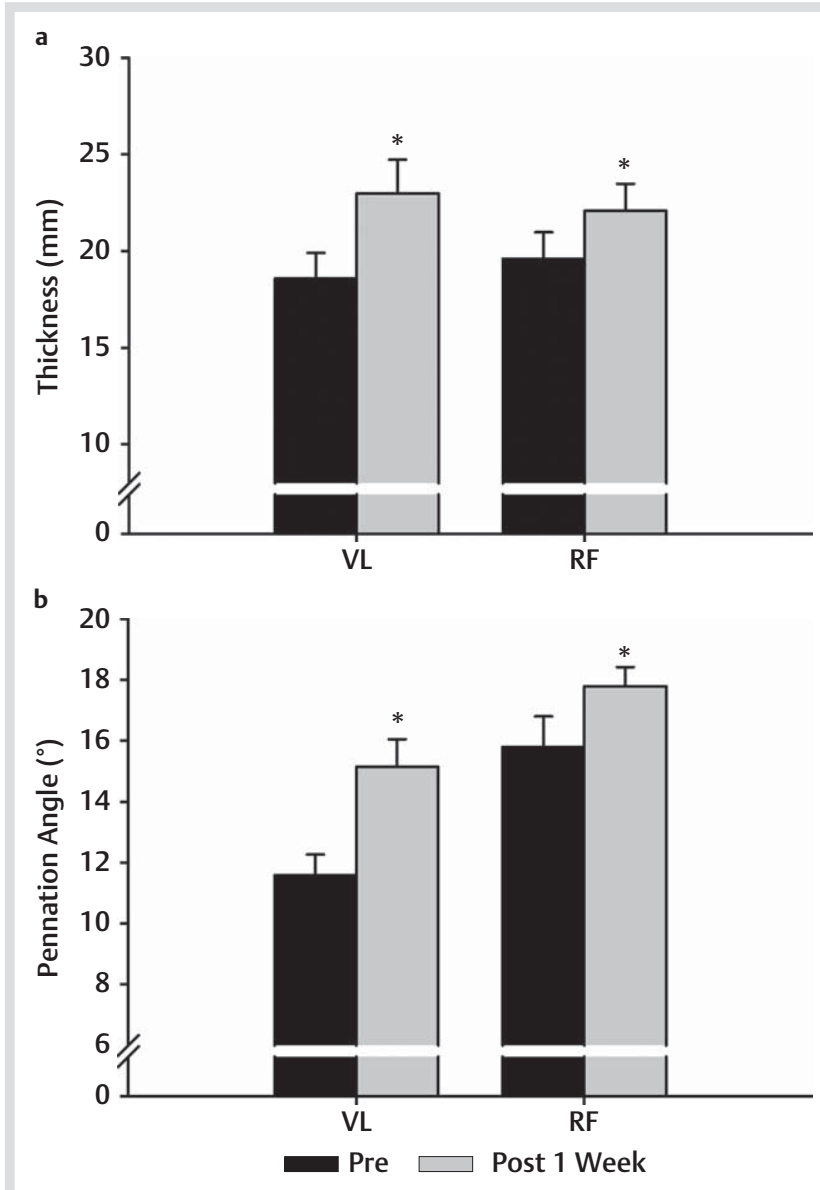

Fig. 4 Pre- to post-training changes in vastus lateralis (VL) and rectus femoris (RF) muscle thickness $\mathbf{a}$ and pennation angles $\mathbf{b}$. Values are presented as mean \pm SEM. ${ }^{*} \mathrm{P}<0.05$ vs. pre-training.

current study was more aggressive than those employed by previous investigators $[8,29]$, our results suggested that our protocol can be safely administered and tolerated by healthy individuals.

To the best of our knowledge, this is the first report of increased maximum cycling power following eccentric cycling training in young healthy populations. Previous investigators using similar protocols reported findings of unchanged concentric cycling power [8]. We believe the most plausible explanation for these differences is the timing of the post-training evaluation of muscular function. The non-significant findings reported by previous investigators may be a reflection of suppressed muscular function due to muscle remodeling following high-intensity eccentric exercise. Several investigators have reported long lasting indications of remodeling with suppressed muscle force after eccentric exercise $[1,33]$. Results from mechanistic studies indicate that a cascade of events resulting in muscle remodeling occur in response to eccentric training [19,25,26,35]. These events include satellite cell activation and proliferation for mediating muscle remodeling during the regenerative process. Furthermore, muscle remodeling can also be initiated independent of discernible damage to the muscle $[14,28]$. Therefore, posttraining measures will accurately reflect optimum muscular function if the time course for the cascade of events that result in complete muscle remodeling is observed in the regenerative process. Indeed, Kadi and colleagues (2004) reported that satellite cells remained significantly elevated at 60 days of detraining
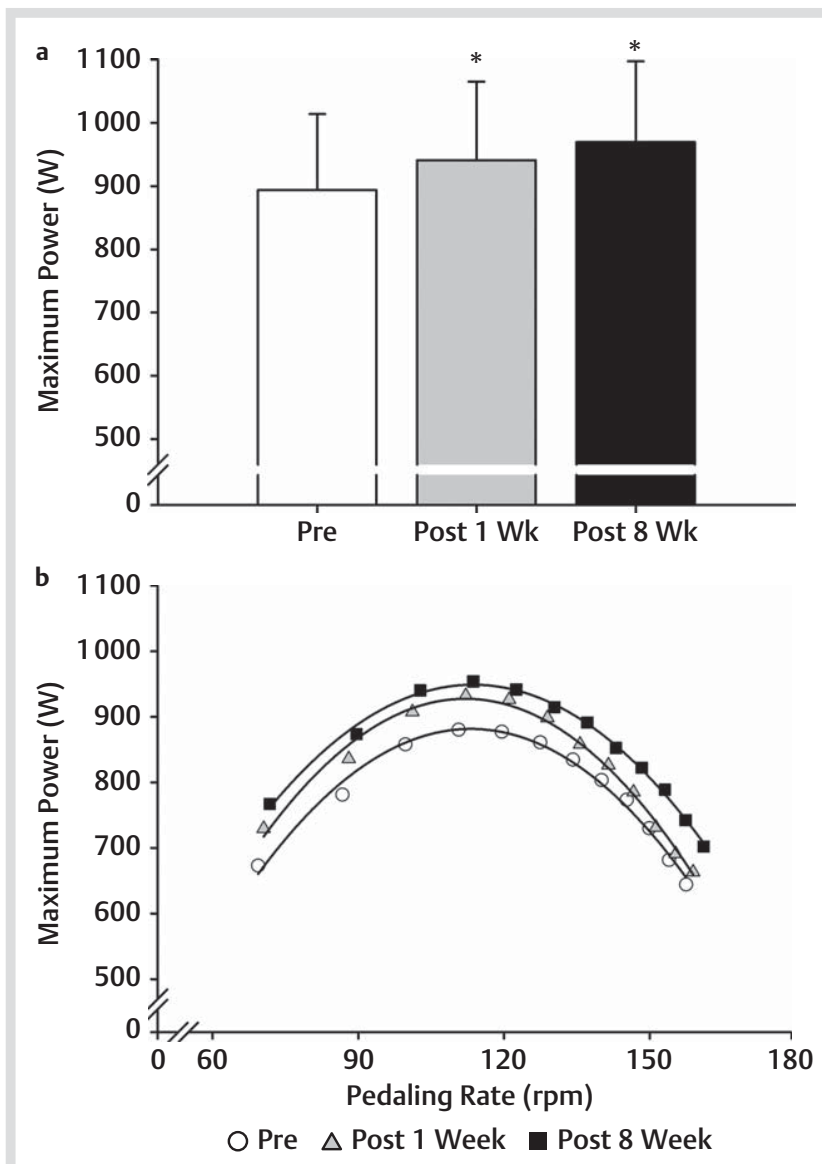

Fig. 5 Pre- to-post-training changes in maximum concentric cycling power a. Complete powerpedaling rate relationships $\mathbf{b}$ were shifted upward at 1 - and 8-weeks post-training. Values are presented as mean \pm SEM. SEM bars in panel $\mathbf{b}$ were removed for clarity. ${ }^{*} \mathrm{P}<0.05$ vs. pre-training value. Note that, maximum power measured at 8 weeks post-training tended to be greater than that measured at 1 week posttraining $(\mathrm{P}=0.06)$.

following heavy resistance training. Hence, the time course of a muscle remodeling event such as satellite cell content modulation could explain the larger increase in maximum concentric cycling power measured at 8 weeks post-training. The present data allow us to speculate that previous investigations evaluating maximum concentric strength and/or power $[8,17]$ might have shown significant increases if additional post-training assessments were performed at a later time point. Such evaluation might have provided time for full muscular recovery, which would allow the positive consequences of eccentric cycling training to emerge.

The results of increased RF and VL muscle thickness and pennation angles demonstrated training-induced alterations in muscle structure. Previous investigators have reported increases in muscle fiber cross-sectional area [29] and lean muscle mass [17] in healthy individuals following eccentric cycling training. Thus, the results from the current investigation relating to muscle structure improvements support and unite these previous findings. Hypertrophy after eccentric cycling training would be expected given the high forces and powers elicited during repetitive eccentric muscle contractions [5]. In addition, an increase in pennation angle provides variable gearing to improve the modulation of force output for contractions against high loads [3] and can also be regarded as a strategy for attaching more 
contractile material along the tendon aponeurosis [10]. Hence, the training-induced hypertrophy and concomitant increase in pennation angle may account for muscular function improvements observed in this investigation. Because logistical issues prevented the assessment of muscle structure at the 8-weeks post-training time point, our present results only allow us to speculate that these muscular adaptations were retained at the 8 -weeks post-training time point. Furthermore, adaptations to strength training have been shown to be retained for at least 31 weeks after cessation of training in young healthy individuals [24]. Neural adaptations associated with the activation of agonistic, synergistic and antagonistic muscles [27] could have also contributed to the improvement in maximum concentric cycling power. A future direction for our laboratory will be to quantify and separate the contributions of muscular and neural adaptations with more direct measures (e.g., electromyography).

An extensive review by Isner-Horobeti et al. (2013) highlighted more than 15 investigations demonstrating that eccentric cycling training is more effective than traditional concentric and/or strength training at improving muscular function in a variety of populations ranging from patients with central limitations to competitive athletes [23]. Based on these previous findings, we did not include a control group in the present study. Despite the improvements in quadriceps muscle structure and maximum cycling power observed in this study, obtaining direct measures of muscle damage, as well as muscular and neural adaptations, will provide a more complete description of the repeated bout effect and the modulation of adaptations associated with chronic eccentric cycling.

In summary, short-duration high-intensity eccentric cycling training resulted in improvements in muscle structure, reflected in improved $\mathrm{P}_{\max }$. These results also suggest that allowing sufficient time for recovery is important for detecting functional gains following eccentric cycling training.

\section{Acknowledgements}

$\nabla$

The authors would like to thank the participants who volunteered in this study and the students in the Neuromuscular Function Laboratory for their assistance with the data collection. The authors would also like to thank Scott Johnson, D.O. for loaning the ultrasound system and assisting in the sonography of the participants. We also extend our appreciation to Drs. Bruce MacWilliams, Barry Shultz, and Charlie Hicks-Little for their insightful comments during the preparation of the manuscript.

\section{References}

1 Allen DG. Eccentric muscle damage: mechanisms of early reduction of force. Acta Physiol Scand 2001; 171: 311-319

2 Avela J, Kyrolainen H, Komi PV, Rama D. Reduced reflex sensitivity persists several days after long-lasting stretch-shortening cycle exercise. J Appl Physiol 1999; 86: 1292-1300

3 Azizi E, Brainerd EL, Roberts TJ. Variable gearing in pennate muscles. Proc Natl Acad Sci USA 2008; 105: 1745-1750

4 Ben-Sira D, Ayalon A, Tavi M. The effect of different types of strength training on concentric strength in women. J Strength Cond Res 1995; 9: $143-148$

5 Booth FW, Tseng BS, Fluck M, Carson JA. Molecular and cellular adaptation of muscle in response to physical training. Acta Physiol Scand 1998; 162: 343-350

6 Clarkson PM, Nosaka K, Braun B. Muscle function after exerciseinduced muscle damage and rapid adaptation. Med Sci Sports Exerc 1992; 24: 512-520
7 Dartnall TJ, Nordstrom MA, Semmler JG. Adaptations in biceps brachii motor unit activity after repeated bouts of eccentric exercise in elbow flexor muscles. J Neurophysiol 2011; 105: 1225-1235

8 Elmer SJ, Hahn S, McAllister P, Leong C, Martin JC. Improvements in multi-joint leg function following chronic eccentric exercise. Scand J Med Sci Sports 2012; 22: 653-661

9 Elmer SJ, Martin JC. Construction of an isokinetic eccentric cycle ergometer for research and training. J Appl Biomech 2012 [Epub ahead of print]

10 Farup J, Kjolhede T, Sorensen H, Dalgas U, Moller AB, Vestergaard PF, Ringgaard S, Bojsen-Moller J, Vissing K. Muscle morphological and strength adaptations to endurance vs. resistance training. J Strength Cond Res 2012; 26: 398-407

11 Faulkner JA, Brooks SV, Opiteck JA. Injury to skeletal muscle fibers during contractions: conditions of occurrence and prevention. Phys Ther 1993; 73: 911-921

12 Field $A$ (ed.). Discovering statistics using SPSS. Los Angeles: SAGE, 2009; 821

13 Fitts RH, McDonald KS, Schluter JM. The determinants of skeletal muscle force and power: their adaptability with changes in activity pattern. J Biomech 1991; 24 (Suppl 1): 111-122

14 Flann KL, LaStayo PC, McClain DA, Hazel M, Lindstedt SL. Muscle damage and muscle remodeling: no pain, no gain? J Exp Biol 2011; 214: 674-679

15 Fukunaga T, Miyatani M, Tachi M, Kouzaki M, Kawakami Y, Kanehisa $H$. Muscle volume is a major determinant of joint torque in humans. Acta Physiol Scand 2001; 172: 249-255

16 Gerber JP, Marcus RL, Dibble LE, Greis PE, Burks RT, LaStayo PC. Effects of early progressive eccentric exercise on muscle structure after anterior cruciate ligament reconstruction. J Bone Joint Surg Am 2007; 89: 559-570

17 Gross M, Luthy F, Kroell J, Muller E, Hoppeler H, Vogt M. Effects of eccentric cycle ergometry in alpine skiers. Int J Sports Med 2010; 31: $572-576$

18 Harriss DJ, Atkinson G. Update - Ethical standards in sport and exercise science research. Int J Sports Med 2011; 32: 819-821

19 Hawke TJ, Garry DJ. Myogenic satellite cells: physiology to molecular biology. J Appl Physiol 2001; 91: 534-551

20 Higbie EJ, Cureton KJ, Warren GL $3^{\text {rd }}$, Prior BM. Effects of concentric and eccentric training on muscle strength, cross-sectional area, and neural activation. J Appl Physiol 1996; 81: 2173-2181

21 Hortobagyi T, Dempsey L, Fraser D, Zheng D, Hamilton G, Lambert J, Dohm $L$. Changes in muscle strength, muscle fibre size and myofibrillar gene expression after immobilization and retraining in humans. J Physiol 2000; 524 (Pt 1): 293-304

22 Hortobagyi T, Hill JP, Houmard JA, Fraser DD, Lambert NJ, Israel RG. Adaptive responses to muscle lengthening and shortening in humans. J Appl Physiol 1996; 80: 765-772

23 Isner-Horobeti ME, Dufour SP, Vautravers P, Geny B, Coudeyre E, Richard $R$. Eccentric exercise training: modalities, applications and perspectives. Sports Med 2013; 43: 483-512

24 Ivey FM, Tracy BL, Lemmer JT, NessAiver M, Metter EJ, Fozard JL, Hurley $B F$. Effects of strength training and detraining on muscle quality: age and gender comparisons. J Gerontol A Biol Sci Med Sci 2000; 55: B152-B157, discussion B158-B159

25 Kadi F, Schjerling P, Andersen LL, Charifi N, Madsen JL, Christensen LR, Andersen JL. The effects of heavy resistance training and detraining on satellite cells in human skeletal muscles. J Physiol 2004; 558: 1005-1012

26 Kadi F, Thornell LE. Concomitant increases in myonuclear and satellite cell content in female trapezius muscle following strength training. Histochem Cell Biol 2000; 113: 99-103

27 Kraemer WJ, Fleck SJ, Evans WJ. Strength and power training: physiological mechanisms of adaptation. Exerc Sport Sci Rev 1996; 24: 363-397

28 Lastayo P, Marcus RL, Dibble L, Frajacomo F, Lindstedt SL. Eccentric exercise in rehabilitation: safety, feasibility and application. J Appl Physiol doi: 10.1152/japplphysiol.00008.2013

29 LaStayo PC, Pierotti DJ, Pifer J, Hoppeler H, Lindstedt SL. Eccentric ergometry: increases in locomotor muscle size and strength at low training intensities. Am J Physiol 2000; 278: R1282-R1288

30 Martin JC, Diedrich D, Coyle EF. Time course of learning to produce maximum cycling power. Int J Sports Med 2000; 21: 485-487

31 Martin JC, Wagner BM, Coyle EF. Inertial-load method determines maximal cycling power in a single exercise bout. Med Sci Sports Exerc 1997; 29: 1505-1512

32 Morrissey MC, Harman EA, Johnson MJ. Resistance training modes: specificity and effectiveness. Med Sci Sports Exerc 1995; 27: 648-660 
33 Proske U, Morgan DL. Muscle damage from eccentric exercise: mechanism, mechanical signs, adaptation and clinical applications. J Physiol 2001; 537: 333-345

34 Raue U, Terpstra B, Williamson DL, Gallagher PM, Trappe SW. Effects of short-term concentric vs. eccentric resistance training on single muscle fiber MHC distribution in humans. Int J Sports Med 2005; 26: 339-343

35 Rosenblatt JD, Yong D, Parry DJ. Satellite cell activity is required for hypertrophy of overloaded adult rat muscle. Muscle Nerve 1994; 17: 608-613
36 Ryan LM, Magidow PS, Duncan PW. Velocity-specific and mode-specific effects of eccentric isokinetic training of the hamstrings. J Orthop Sports Phys Ther 1991; 13: 33-39

37 Sayers SP, Clarkson PM. Force recovery after eccentric exercise in males and females. Eur J Appl Physiol 2001; 84: 122-126

38 Woltring HJ. A Fortran package for generalized, cross-validatory spline smoothing and differentiation. Adv Eng Softw 1986; 8: 104-113 